\title{
A NEW SPECTRAL THEORY FOR NONLINEAR OPERATORS AND ITS APPLICATIONS
}

\author{
W. FENG
}

\begin{abstract}
In this paper, by applying $(p, k)$-epi mapping theory, we introduce a new definition of spectrum for nonlinear operators which contains all eigenvalues, as in the linear case. Properties of this spectrum are given and comparison is made with the other definitions of spectra. We also give applications of the new theory.
\end{abstract}

\section{INTRODUCTION}

Spectral theories for nonlinear operators have been extensively studied, for example, see [1], [5]-[7] and [9]. Different attempts have been made to define the spectrum for nonlinear operators. Clearly, a good definition should preserve as many properties of the spectrum for classical bounded linear operators as possible and reduce to the familiar spectrum in the case of linear operators. In [15], a spectrum for Lipschitz continuous operators was studied (we denote it by $\sigma_{l i p}(f)$ ), which is compact but may be empty (see the example in Section 5). The spectrum introduced by Furi, Martelli and Vignoli (denoted $\sigma_{f m v}(f)$ ) has found many interesting applications (see [9]). However, it was indicated in [6] that this spectrum may be disjoint from the eigenvalues, which is an important part of the spectrum in the linear case. This paper is mainly based on the study of [9] and the main aim is to introduce a new spectrum for nonlinear operators by applying the $(p, k)$-epi mapping theory of [10], [14], [17]. This new version of the spectrum is closed and contains all the eigenvalues as in the case of linear operators.

This paper is organized as follows. In Section 3, the definition of the new spectrum will be given and some of its special properties will be proved. For example, we will show that this spectrum is closed, bounded, upper

1991 Mathematics Subject Classification. Primary 47H12.

Key words and phrases. Spectrum of a nonlinear operator, eigenvalues, $(p, k)$-epi mapping theory.

Received: September 1, 1997. 
semicontinuous and contains all the eigenvalues. In Section 4, we investigate positively homogeneous operators where more precise results are possible. Some of the results are direct generalizations of the spectral theory for linear operators. The results in this section will be used in Section 6 to generalize some existence theorems. In Section 5, we will compare our spectrum $\sigma(f)$ with the two spectra mentioned before $\left(\sigma_{f m v}(f)\right.$ and $\left.\sigma_{\text {lip }}(f)\right)$. We will prove that, in general,

$$
\sigma_{\text {lip }}(f) \supsetneqq \sigma(f) \supsetneqq \sigma_{f m v}(f) .
$$

Moreover, in this section, nonemptiness of spectra is discussed. A counterexample shows that all these spectra may be empty, which answers one of the open questions in [15].

In the last section, some applications of the new theory are obtained. This theory enables us to generalize the Birkoff-Kellogg theorem and the Hopf theorem on spheres, which were also consequences of the theory in [9]. A non-trivial existence result for a global Cauchy problem, which was studied in [14], is obtained by applying the new theory.

It would be interesting to see extensions of the present theory to the cases considered by Ding and Kartsatos in [4], where the concept of a $(p, 0)$ epi mapping of [10] has been extended to cover perturbations of (possibly) densely defined operators.

In Section 2, we give some notations and definitions which will be used in the sequel.

\section{Preliminaries}

Let $E$ and $F$ be complex Banach spaces and $\Omega$ be an open bounded subset of $E$. We suppose that $f: E \rightarrow E$ is continuous and $\alpha(A)$ denotes the measure of noncompactness of a bounded set $A[3]$. The following notations will be used in the sequel.

$$
\begin{aligned}
\alpha(f) & =\inf \{k \geq 0: \alpha(f(A)) \leq k \alpha(A) \text { for every bounded } A \subset E\}, \\
\omega(f) & =\sup \{k \geq 0: \alpha(f(A)) \geq k \alpha(A) \text { for every bounded } A \subset E\}, \\
m(f) & =\sup \{k \geq 0:\|f(x)\| \geq k\|x\| \text { for all } x \in E\}, \\
d(f) & =\liminf _{\|x\| \rightarrow \infty} \frac{\|f(x)\|}{\|x\|}, \quad|f|=\limsup _{\|x\| \rightarrow \infty} \frac{\|f(x)\|}{\|x\|} .
\end{aligned}
$$

Here $|f|$ is called the quasinorm of $f$ and $f$ is said to be quasibounded if $|f|<\infty$. Maps with $\alpha(f)<1$ are $k$-set contractive (also condensing) with $k=\alpha(f)$. Note that a map $f$ satisfies $\alpha(f)=0$ if and only if $f$ is compact, that is $\overline{f(A)}$ is compact for every bounded set $A$. For the properties of $\alpha(f), \omega(f)$ and $d(f)$ we refer to [9].

A map $f: E \rightarrow F$ is said to be stably-solvable if given any compact map $h: E \rightarrow F$ with zero quasinorm, there is at least one element $x$ of $E$ such that $f(x)=h(x)$. Spectra $\sigma_{f m v}(f)$ and $\sigma_{l i p}(f)$ are defined as follows. 
Definition 2.1. (see [9]) $f$ is said to be fmv-regular if it is stably-solvable and $d(f)$ and $\omega(f)$ are both positive. Let

$$
\rho_{f m v}(f)=\{\lambda \in \mathbb{C}: \lambda I-f \text { is } f m v-\text { regular }\},
$$

then $\sigma_{f m v}(f)=\mathbb{C} \backslash \rho_{f m v}(f)$.

Definition 2.2. (see [15]) Let $\operatorname{Lip}(E)$ be the space of all Lipschitz mappings. For $A \in \operatorname{Lip}(E)$, the Lip-spectrum is defined by

$$
\sigma_{\text {lip }}(A)=\left\{\lambda:(\lambda I-A)^{-1} \text { does not exists or }(\lambda I-A)^{-1} \notin \operatorname{Lip}(E)\right\} .
$$

The $p$-epi mappings were introduced by Furi, Martelli and Vignoli [10] and then were studied and applied in [12], [14], [17]. In [17], the notion was generalized to the following $(p, k)$-epi mappings.

Definition 2.3. A continuous mapping $f: \bar{\Omega} \rightarrow F$ is said to be $p$-admissible $(p \in F)$ if $f(x) \neq p$ for $x \in \partial \Omega$.

A 0 -admissible mapping $f: \bar{\Omega} \rightarrow F$ is said to be $(0, k)$-epi if for each $k$-set contraction $h: \bar{\Omega} \rightarrow F$ with $h(x) \equiv 0$ on $\partial \Omega$ the equation $f(x)=h(x)$ has a solution in $\Omega$. Similarly, a $p$-admissible mapping $f: \bar{\Omega} \rightarrow F$ is said to be $(\mathrm{p}, \mathrm{k})$-epi if the mapping $f-p$ defined by $(f-p)(x)=f(x)-p, x \in \bar{\Omega}$, is $(0, k)$-epi.

It was shown in [17] that the $(p, k)$-epi mappings have similar properties with properties usually obtained via the topological degree, for example, the homotopy property and boundary dependence property.

In the following, let $B_{r}=\{x: x \in E,\|x\| \leq r\}$ and $\partial B_{r}$ denote the boundary of $B_{r}$. If for every $x \neq 0, f(x) \neq 0$, let

$$
\begin{aligned}
\nu_{r}(f, 0)= & \inf \left\{k \geq 0, \text { there exists } g: B_{r} \rightarrow E, \text { with } \alpha(g) \leq k,\right. \\
& \left.g \equiv 0 \text { on } \partial B_{r} \text { s.t. } f(x)=g(x) \text { has no solutions in } B_{r}\right\},
\end{aligned}
$$

and $\nu(f)=\inf \left\{\nu_{r}(f, 0), r>0\right\}$. We will call $\nu(f)$ the measure of solvability of $f$ at 0 . (This concept is related to the measure of unsolvability of $f$ at $p$, which was introduced in [17]). Notice that, $\nu(f)>0$ if and only if there exists $\varepsilon>0$, such that $f(x)$ is $(0, \varepsilon)$-epi on every $B_{r}$ with $r>0$.

\section{A NEW DEFinition OF THE SPECTRUm FOR CONTINUOUS OPERATORS}

We begin with the following definition.

Definition 3.1. Suppose that $f: E \rightarrow E$ is continuous, then $f$ is said to be regular if

$$
\omega(f)>0, \quad m(f)>0, \quad \text { and } \quad \nu(f)>0 .
$$

For $\lambda \in \mathbb{C}$, if $\lambda I-f$ is regular, $\lambda$ is said to be in the resolvent set of $f$. Let $\rho(f)$ denote the resolvent set of $f$, then the spectrum of $f$ is defined as follows:

$$
\sigma(f)=\{\lambda \in \mathbb{C}: \lambda I-f \text { is not regular }\}=\mathbb{C} \backslash \rho(f) .
$$

Proposition 3.2. If $f$ is a regular map, then $f$ is surjective. 
Proof. Since $f$ is regular, $m(f)>0$. Thus for $x \in E,\|f(x)\| \rightarrow \infty$ as $\|x\| \rightarrow \infty$. Also we have $\nu(f)>0$, so there exists $\varepsilon>0$ such that $f$ is $(0, \varepsilon)$-epi on every $B_{r}$ with $r>0$. By Corollary 3.2 of [17], $f$ is surjective.

The following theorem characterizes the regular maps among continuous linear operators.

Theorem 3.3. Suppose that $E$ is a normed linear space, $f: E \rightarrow E$ is a continuous linear operator. Then $f$ is regular if and only if $f$ is a linear homeomorphism.

Proof. Assume that $f$ is regular. By Proposition 3.2, $f$ is surjective. Also, $m(f)>0$ implies that $f$ is one to one and $\left\|f^{-1}(x)\right\| \leq(1 / m(f))\|x\|$. Thus $f^{-1}$ is continuous, so $f$ is a linear homeomorphism.

Conversely, suppose that $f$ is a linear homeomorphism. Then $f^{-1}$ is a bounded linear operator and for all $x \in E,\|f(x)\| \geq\left(1 /\left\|f^{-1}\right\|\right)\|x\|$. This ensures that

$$
m(f) \geq 1 /\left\|f^{-1}\right\|, \quad \omega(f) \geq 1 /\left\|f^{-1}\right\| .
$$

So for $0<\varepsilon<1 /\left\|f^{-1}\right\|, f$ is $(0, \varepsilon)$-epi on every $B_{r}$ with $r>0$ [17]. Thus $\nu(f)>\varepsilon>0$, and $f$ is regular.

Remark 3.4. By Theorem 3.3, for a bounded linear operator $f$, the spectrum of $f$ in Definition 3.1 is the same as the usual one.

It is well known in linear spectral theory that $\sigma(f)$ is closed and $\rho(f)$ is open. The following theorem shows that this property holds true for the spectrum of nonlinear maps given by Definition 3.1.

Theorem 3.5. For a continuous map $f, \rho(f)$ is an open set and $\sigma(f)$ is closed.

Proof. Suppose that $\lambda \in \rho(f)$, then

$$
\omega(\lambda I-f)>0, m(\lambda I-f)>0,
$$

and $\lambda I-f$ is $(0, \varepsilon)$-epi on every $B_{r}$ with $r>0$ for some $\varepsilon>0$. Now let

$$
\delta_{1}=\omega(\lambda I-f) / 2, \delta_{2}=m(\lambda I-f) / 2, \delta_{3}=\varepsilon / 2,
$$

and $\delta=\min \left\{\delta_{1}, \delta_{2}, \delta_{3}\right\}$. Assume that $\mu \in \mathbb{C},|\mu-\lambda|<\delta$. We shall prove that $\mu \in \rho(f)$. Since

$$
|\omega(\mu I-f)-\omega(\lambda I-f)| \leq \alpha(\mu I-\lambda I)=|\mu-\lambda|<\omega(\lambda I-f) / 2,
$$

we have

$$
\omega(\mu I-f)>\omega(\lambda I-f) / 2>0 .
$$

For every $x \in E$,

$$
\|\mu x-f(x)\| \geq\|\lambda x-f(x)\|-|\mu-\lambda|\|x\| \geq(m(\lambda I-f) / 2)\|x\|,
$$

so

$$
m(\mu I-f) \geq m(\lambda I-f) / 2 .
$$


Furthermore, let $h:[0,1] \times E \rightarrow E$ be defined by $h(t, x)=t(\mu-\lambda) I$. Then $h$ is a $(\mu-\lambda)$-set contraction. Let

$$
S=\{x \in E: \lambda x-f(x)+t(\mu-\lambda) x=0 \text { for some } t \in(0,1]\} .
$$

Then for every $x \in S$,

$$
\|\lambda x-f(x)\|=\|t(\mu-\lambda) x\| \geq m(\lambda I-f)\|x\| .
$$

Hence

$$
|\mu-\lambda|\|x\| \geq m(\lambda I-f)\|x\| .
$$

Thus $x=0$ and $S=\{0\}$. By the $(0, k)$-epi Homotopy-property (see [17]), $\mu I-f$ is $(0, \varepsilon-|\mu-\lambda|)$-epi on every $B_{r}$ with $r>0$. So

$$
\nu(\mu I-f)>\varepsilon-|\mu-\lambda|>\varepsilon / 2>0 .
$$

Therefore, $\mu \in \rho(f)$.

We recall that for a bounded linear operator, its spectrum is always bounded. The following theorem generalizes this result to the nonlinear case.

Theorem 3.6. Let $E$ be a Banach space and $f: E \rightarrow E$ be a continuous map. Assume that $\alpha(f)<\infty$ and there exists a constant $M>0$ such that $\|f(x)\| \leq M\|x\|$ for all $x \in E$. Then $\sigma(f)$ is bounded.

Proof. Let $\lambda \in \mathbb{C}$ with $|\lambda|>\max \{M, \alpha(f)\}$, we shall prove that $\lambda \in \rho(f)$. Firstly, by Proposition 3.1.3 of [9] we have

$$
\omega(\lambda I-f) \geq|\lambda|-\alpha(f)>0 .
$$

Also, for every $x \in E$, the inequality

$$
\|(\lambda I-f)(x)\| \geq(|\lambda|-M)\|x\|
$$

implies that $m(\lambda I-f)>0$. Now let $\varepsilon>0$ be such that $\alpha(f)+\varepsilon<|\lambda|$, we shall show that $\lambda I-f$ is $(0, \varepsilon)$-epi on every $B_{r}$ with $r>0$. Let

Suppose $h$ is a $\alpha$-lipschitz map with constant $\varepsilon$, and $h(x)=0$ for $x \in \partial B_{r}$.

$$
h_{1}(x)= \begin{cases}h(x) & \text { for }\|x\| \leq r, \\ 0 & \text { for }\|x\|>r .\end{cases}
$$

$h_{1}$ is continuous on $E$. For every bounded subset $A \subset E$,

$$
\begin{aligned}
\alpha\left(h_{1}(A)\right) & =\alpha\left(h_{1}\left(A \cap B_{r}\right)\right) \\
& =\alpha\left(h\left(A \cap B_{r}\right)\right) \\
& \leq \varepsilon \alpha\left(A \cap B_{r}\right) \leq \varepsilon \alpha(A) .
\end{aligned}
$$

Hence $h_{1}$ is also an $\alpha$-Lipschitz map with constant $\varepsilon$. Let

$$
S=\left\{x: x-t f(x) / \lambda=h_{1}(x) / \lambda, \text { for some } t \in[0,1]\right\} .
$$

For all $x$ with $\|x\| \geq r$ we have $h_{1}(x)=0$ and

$$
\|\lambda(x-t f(x) / \lambda)\| \geq|\lambda|\|x\|-\|f(x)\| \geq(|\lambda|-M)\|x\|>0 .
$$

This implies that $S \subset B_{r}$. Since $h_{1} / \lambda$ is a $\varepsilon /|\lambda|$-set contraction, $\varepsilon /|\lambda|<1$, and $h_{1}(x) / \lambda \equiv 0$ on $\partial B_{r}$, the fact that $I$ is $(0, \varepsilon)$-epi for all $\varepsilon<1$ implies that 
the equation $x=h_{1}(x) / \lambda$ has a solution in $B_{r}$. Thus $S \neq \emptyset$ and $S \cap \partial B_{r}=\emptyset$. Next we have

$$
S \subset[0,1] f(S) / \lambda+h_{1}(S) / \lambda
$$

Therefore,

$$
\alpha(S) \leq((\alpha(f)+\varepsilon) /|\lambda|) \alpha(S) .
$$

Hence $S$ is compact because $\alpha(f)+\varepsilon<|\lambda|$ and $S$ is closed. Let $\phi$ be a continuous function such that $0 \leq \phi \leq 1$ and

$$
\phi(x)= \begin{cases}1 & \text { for } x \in S \\ 0 & \text { for }\|x\| \geq r\end{cases}
$$

and let

$$
g(x)=\phi(x) f(x) / \lambda+h_{1}(x) / \lambda .
$$

Then $g$ is an $(\alpha(f)+\varepsilon) /|\lambda|$-set contraction, $g(x) \equiv 0$ on the boundary of $B_{r}$. Hence $x=g(x)$ has a solution $x_{0} \in B_{r}$. Then $x_{0} \in S$ so $\phi\left(x_{0}\right)=1$ and $h_{1}\left(x_{0}\right)=h\left(x_{0}\right)$. Thus $x_{0}$ is a solution of the equation

$$
x-f(x) / \lambda=h(x) / \lambda .
$$

This ensures that $\lambda I-f$ is $(0, \varepsilon)$-epi on $B_{r}$, so we have $\nu(f) \geq \varepsilon>0, \lambda I-f$ is regular, and $\lambda$ is in the resolvent set of $f$.

Remark 3.7. For nonlinear map $f$ with $f(0)=0$, we define the norm of $f$ by

$$
\|f\|=\inf \{k>0:\|f(x)\| \leq k\|x\|\} .
$$

Defining the radius of the spectrum of $f$ by $r_{\sigma}(f)=\sup \{|\lambda|: \lambda \in \sigma(f)\}$, it follows that

$$
r_{\sigma}(f) \leq \max \{\alpha(f),\|f\|\}
$$

If $f(0) \neq 0$, for each $\lambda \in \mathbb{C}$, either $\lambda I-f$ is not surjective, or there exists $x \in E, x \neq 0$, such that $\lambda x-f(x)=0$, and then $\lambda$ is a eigenvalue of $f$. By the following theorem, in both these cases, $\lambda \in \sigma(f)$. Thus $\sigma(f)=\mathbb{C}$. Hence in what follows, unless otherwise stated, we shall assume that $f(0)=0$.

Theorem 3.8. All the eigenvalues of $f$ are in the spectrum of $f$.

Proof. If $f(x)=\lambda x$ with $x \neq 0$, then $m(\lambda I-f)=0$, so $\lambda \in \sigma(f)$.

As mentioned in Section 1, the above simple theorem represents the big difference between $\sigma_{f m v}(f)$ and Definition 3.1. According to their definition, the spectrum may be disjoint with its eigenvalues [6], but it is well known that for a linear operator, one of the important parts of its spectrum is the point spectrum, the eigenvalues.

The following lemma enables us to prove the upper semicontinuity of the spectrum.

Lemma 3.9. Let $A \subset \mathbb{K}(\mathbb{K}=\mathbb{C}$ or $\mathbb{R})$ be compact with $A \cap \sigma(f)=\emptyset$. Then there exists $\varepsilon>0$ such that for $\mu \in A$ and $g: E \rightarrow E$, a continuous map with $\|f-g\|<\varepsilon, \alpha(f-g)<\varepsilon$, it follows that $\mu \notin \sigma(g)$, where

$$
\|f-g\|=\inf \{k \geq 0:\|f(x)-g(x)\| \leq k\|x\|, x \in E\} .
$$


Proof. For $\lambda \in A$, we have

$$
\omega(\lambda I-f)>0, \quad m(\lambda I-f)>0, \quad \text { and } \quad \nu(\lambda I-f)>0 .
$$

Thus $\lambda I-f$ is $\left(0, \varepsilon_{0}\right)$-epi for some $\varepsilon_{0}>0$ on every $B_{r}$. By the proof of Theorem 3.5, there exists $\delta_{\lambda}>0$ such that for every $\lambda^{\prime}$ with $\left|\lambda^{\prime}-\lambda\right|<\delta_{\lambda}$,

$$
\omega\left(\lambda^{\prime} I-f\right)>\omega(\lambda I-f) / 2, \quad m\left(\lambda^{\prime} I-f\right)>m(\lambda I-f) / 2,
$$

and $\lambda^{\prime} I-f$ is $\left(0, \varepsilon_{0} / 2\right)$-epi on $B_{r}$. Let

$$
0<\varepsilon_{\lambda}<\min \left\{\omega(\lambda I-f) / 2, m(\lambda I-f) / 2, \varepsilon_{0} / 2\right\},
$$

and assume that $\|g-f\|<\varepsilon_{\lambda}, \alpha(g-f)<\varepsilon_{\lambda}$. Then by Proposition 3.1.3 of $[9]$

and

$$
\omega\left(\lambda^{\prime} I-g\right) \geq \omega\left(\lambda^{\prime} I-f\right)-\alpha(f-g)>0,
$$

$$
\left\|\left(\lambda^{\prime} I-g\right)(x)\right\| \geq\left\|\lambda^{\prime} x-f(x)\right\|-\|f(x)-g(x)\|>\left(m(\lambda I-f) / 2-\varepsilon_{\lambda}\right)\|x\| .
$$

Hence $m\left(\lambda^{\prime} I-g\right)>0$. Furthermore, for every $t \in(0,1]$, and $x \neq 0$,

$$
\left\|\lambda^{\prime} x-f(x)+t(f(x)-g(x))\right\|>0 .
$$

By the Continuation Principle for $(0, k)$-epi mappings [14], $\lambda^{\prime} I-g$ is $\left(0, r_{0}\right)$ epi for each $r_{0}>0$ with

$$
r_{0}<\min \left\{(\omega(\lambda I-f) / 2)-\alpha(f-g),\left(\varepsilon_{0} / 2\right)-\alpha(f-g)\right\} .
$$

This implies that $\nu\left(\lambda^{\prime} I-g\right)>0$, so $\lambda^{\prime} \in \rho(g)$. Let

$$
O\left(\lambda, \delta_{\lambda}\right)=\left\{\lambda^{\prime} \in \mathbb{K}:\left|\lambda^{\prime}-\lambda\right|<\delta_{\lambda}\right\},
$$

the above discussion implies that $\bigcup_{\lambda \in A} O\left(\lambda, \delta_{\lambda}\right) \supset A$. Since $A$ is compact, there exist a finite collection such that $\bigcup_{i=1}^{n} O\left(\lambda_{i}, \delta_{\lambda_{i}}\right) \supset A$. Let $\varepsilon=\min \left\{\varepsilon_{\lambda_{1}}, \varepsilon_{\lambda_{2}}, \cdots, \varepsilon_{\lambda_{n}}\right\}$, and suppose that $\|g-f\|<\varepsilon, \alpha(g-f)<\varepsilon$. For $\mu \in A$, if $\mu \in O\left(\lambda_{i}, \delta_{\lambda_{i}}\right), i \in\{1, \cdots, n\}$, then

$$
\|g-f\|<\varepsilon_{\lambda_{i}}, \alpha(g-f)<\varepsilon_{\lambda_{i}}
$$

imply that $\mu \notin \sigma(g)$.

The following theorem whose proof follows that of Theorem 8.3.2 [9] concerns the upper semicontinuity of the spectrum. We omit its proof here.

Theorem 3.10. Let

$$
\begin{aligned}
p(E)= & \{f: \alpha(f)<+\infty, \text { there exists } M>0 \text { such that }\|f(x)\| \leq M\|x\| \text { for } \\
& x \in E\} .
\end{aligned}
$$

The multivalued map $\sigma: p(E) \rightarrow \mathbb{K}$ which assigns to each $f$ its spectrum $\sigma(f)$, is upper semicontinuous (with compact values).

Suppose that $f(0)=0$, we recall that a point $\lambda$ is called a bifurcation point of $f$ if there are sequences $\lambda_{n} \in \mathbb{K}$ and $x_{n} \in E$ such that $x_{n} \neq$ $0, f\left(x_{n}\right)=\lambda_{n} x_{n}, \lambda_{n} \rightarrow \lambda, x_{n} \rightarrow 0$ as $n \rightarrow \infty$. $\lambda$ is called an asymptotic bifurcation point of $f$ if there are sequences $\lambda_{n} \in \mathbb{K}$ and $x_{n} \in E$ such that $x_{n} \neq 0, f\left(x_{n}\right)=\lambda_{n} x_{n}, \lambda_{n} \rightarrow \lambda,\left\|x_{n}\right\| \rightarrow \infty$ as $n \rightarrow \infty$. The following proposition gives the relation between the spectrum and bifurcation points. 
Proposition 3.11. Bifurcation points and asymptotic bifurcation points of $f$ are in the spectrum of $f$.

Proof. Suppose that $\lambda_{n} \in \mathbb{K}$ and $x_{n} \in E$ are such that $\lambda_{n} \rightarrow \lambda, x_{n} \neq$ $0, \quad\left\|x_{n}\right\| \rightarrow 0$ and $f\left(x_{n}\right)=\lambda_{n} x_{n}$. Then $m(\lambda I-f)=0$. Otherwise the inequality $\left\|\lambda x_{n}-f\left(x_{n}\right)\right\| \geq m(\lambda I-f)\left\|x_{n}\right\|$ would implies that $\left|\lambda-\lambda_{n}\right| \geq$ $m(\lambda I-f)>0$, a contradiction. Hence $\lambda \in \sigma(f)$.

Similarly, if $\lambda$ is an asymptotic bifurcation point of $f$, we obtain $\lambda \in$ $\sigma(f)$.

The following properties of the spectrum are easily checked.

Proposition 3.12. Let $E$ be a normed space and $f: E \rightarrow E$ be a continuous operator. Then for every $\lambda \in \mathbb{K}$,

(1) $\sigma(\lambda f) \equiv \lambda \sigma(f), \quad \sigma(0)=0, \quad \sigma(I)=1, \quad \sigma(\lambda I)=\lambda$.

(2) $\sigma(\lambda I+f) \equiv \lambda+\sigma(f)$.

We close this section with the following proposition devoted to the the study of the nonlinear resolvent.

Proposition 3.13. Assume that $A: E \rightarrow E$ is continuous and $\lambda, \mu \in \rho(A)$. Let

$$
R_{A}(\lambda)=(A-\lambda I)^{-1}, \quad R_{A}(\mu)=(A-\mu I)^{-1}
$$

be the multivalued maps. Then

$$
R_{A}(\lambda) x \subset R_{A}(\mu)\left(I+(\lambda-\mu) R_{A}(\lambda)\right) x, \quad x \in E .
$$

If $\mu I-A$ is injective, then

$$
R_{A}(\lambda) x=R_{A}(\mu)\left(I+(\lambda-\mu) R_{A}(\lambda)\right) x, \quad x \in E .
$$

Proof. Let $y \in R_{A}(\lambda) x$, so that $(A-\lambda I) y=x$. Then we can write $A y-\mu y=$ $x+(\lambda-\mu) y$, so that

$$
y \in(A-\mu I)^{-1}(x+(\lambda-\mu) y) \subset(A-\mu I)^{-1}\left(x+(\lambda-\mu) R_{A}(\lambda) x\right) .
$$

This implies that

$$
R_{A}(\lambda) x \subset R_{A}(\mu)\left(I+(\lambda-\mu) R_{A}(\lambda)\right) x .
$$

When $\mu I-A$ is injective, it is easy to show that equality holds.

\section{Positively homogeneous operators}

According to our new definition, some special properties of eigenvalues in the spectrum of a positively homogeneous operator can be obtained, which will be useful in Section 6. Firstly, we shall prove the following lemmas on the positively homogeneous $(0, k)$-epi mappings from Banach space $E$ to $F$, which will be used later.

Lemma 4.1. Suppose that $f: E \rightarrow F$ is a positively homogeneous mapping and $f$ is $(0, \varepsilon)$-epi on some $B_{r}$ for some $\varepsilon>0$. Then $f$ is $(0, \varepsilon)$-epi on every $B_{R}$ with $R>0$. 
Proof. $f$ is $(0, \varepsilon)$-epi on $B_{r}$ and $f$ is positively homogeneous ensure that $f(x) \neq 0$ for all $x \neq 0$. Thus $f$ is 0 -admissible on $B_{R}$. Assume that $h: E \rightarrow$ $F$ is an $\varepsilon$-set contraction with $h(x)=0$ for $x \in \partial B_{R}$. Let

$$
h_{1}(x)=\frac{r}{R} h\left(\frac{R}{r} x\right) \text {. }
$$

Then for every bounded set $A \subset E$,

$$
\begin{aligned}
\alpha\left(h_{1}(A)\right) & =\frac{r}{R} \alpha\left(h\left(\frac{R}{r} A\right)\right) \\
& \leq \frac{r}{R} \varepsilon \alpha\left(\frac{R}{r} A\right) \\
& =\varepsilon \alpha(A) .
\end{aligned}
$$

So $h_{1}$ is an $\varepsilon$-set contraction too. Furthermore, $h_{1}(x)=0$ for $x \in \partial B_{r}$. Thus the equation

$$
f(x)=\frac{r}{R} h\left(\frac{R}{r} x\right)
$$

has at least one solution $x_{0} \in E$ and $\left\|x_{0}\right\|<r$. Then $(R / r) x_{0} \in B_{R}$ is a solution of the equation $f(x)=h(x)$.

Remark 4.2. Lemma 4.1 and the Localization property of $(0, k)$-epi maps [17] show that a positively homogeneous mapping $f$ is $(0, \varepsilon)$-epi on $\overline{\Omega_{1}}$, where $\Omega_{1} \supset f^{-1}(0)$ and $\Omega_{1}$ is an open bounded set of $E$, if and only if $f$ is $(0, \varepsilon)$-epi on the closure of all the bounded open sets $\Omega \supset f^{-1}(0)$. This is not true if $f$ is not positively homogeneous as the following example shows. Let $f: \mathbb{R} \rightarrow \mathbb{R}$ be the function $f(x)=x^{2}-1$, and let $\Omega_{1}=(-2,-1 / 2) \cup(1 / 2,2)$. Then $f$ is $(0, k)$-epi for every $k \geq 0$ on $\overline{\Omega_{1}}$, but $f$ is not 0 -epi on $(-n, n)$ for $n>2$.

Lemma 4.3. Suppose $f: E \rightarrow F$ is positively homogeneous, $\omega(f)>0$ and $f$ is $(0, \varepsilon)$-epi on $B_{r}$ with $r>0$. Then for each $p \in F$, there exists $R>0$ such that $f$ is $\left(p, \varepsilon_{1}\right)$-epi on $B_{R}$ for some $\varepsilon_{1}>0$.

Proof. Let $p \in F$ and $g(x)=f(x)-p$, then $\alpha(g-f)=0$. Let

$$
S=\{x: f(x)+t(g(x)-f(x))=f(x)-t p=0 \text { for some } t \in(0,1]\} .
$$

We shall show that $S$ is bounded. Otherwise, there would exist a sequence $\left\{x_{n}\right\}_{n=1}^{\infty} \subset S$ with $\left\|x_{n}\right\| \rightarrow \infty$ as $n \rightarrow \infty$. Let $t_{n} \in(0,1]$ be such that $f\left(t_{n}\right)=t_{n} p$. Then

$$
f\left(r x_{n} /\left\|x_{n}\right\|\right)=r t_{n} p /\left\|x_{n}\right\| \rightarrow 0 \text { as } n \rightarrow \infty .
$$

Letting $u_{n}=x_{n} /\left\|x_{n}\right\|$, we would have

$$
\omega(f) \alpha\left(\cup_{n=1}^{\infty} r u_{n}\right) \leq \alpha\left(\cup_{n=1}^{\infty} f\left(r u_{n}\right)\right)=0 .
$$

Since $\omega(f)>0$, we have $\alpha\left(\cup_{n=1}^{\infty} r u_{n}\right)=0$. This implies that there exists a subsequence $r u_{n_{k}} \rightarrow u_{0}$ and $\left\|u_{0}\right\|=r$. Thus

$$
\lim _{k \rightarrow \infty} f\left(r u_{n_{k}}\right)=f\left(u_{0}\right)=0 .
$$

This contradicts the fact that $f$ is 0 -admissible on $B_{r}$. 
Now, let $R>0$ be such that $S \subset B_{R}$. Then $\partial B_{R} \cap S=\emptyset$. By Lemma $4.1, f$ is $(0, \varepsilon)$-epi on $B_{R}$. So, the Continuation principle of $(0, k)$-epi maps [14] ensures that $g$ is at least $\left(0, \varepsilon_{1}\right)$-epi for $0<\varepsilon_{1}<\omega(f)$ and $\varepsilon_{1} \leq \varepsilon$. Thus $f$ is $\left(p, \varepsilon_{1}\right)$-epi on $B_{R}$.

Our next theorem characterizes regular maps among positively homogeneous maps.

Theorem 4.4. Let $f$ be positively homogeneous. Then $f$ is regular if and only if

1. $\omega(f)>0$;

2. There exists $\varepsilon>0$ such that $f$ is $(0, \varepsilon)$-epi on $B_{1}$.

Proof. Clearly, we only need to prove that if $f$ satisfies 1 and 2 , then $f$ is regular.

Suppose that $f$ satisfies 1 and 2 , then by Lemma $4.1, f$ is $(0, \varepsilon)$-epi on every $B_{r}$ with $r>0$. So $\nu(f)>0$. Assume that $m(f)=0$. Then there would exist a sequence $\left\{x_{n}\right\}_{n=1}^{\infty} \in E, x_{n} \neq 0$ such that $\left\|f\left(x_{n}\right)\right\|<(1 / n)\left\|x_{n}\right\|$. Let $u_{n}=x_{n} /\left\|x_{n}\right\|$, then $f\left(u_{n}\right) \rightarrow 0$ as $n \rightarrow \infty$. Moreover,

$$
\omega(f) \alpha\left(\cup_{n=1}^{\infty} u_{n}\right) \leq \alpha\left(\cup_{n=1}^{\infty} f\left(u_{n}\right)\right) .
$$

Hence $\alpha\left(\cup_{n=1}^{\infty} u_{n}\right)=0$. This implies that $\left\{u_{n}\right\}_{n=1}^{\infty}$ has a convergent subsequence $u_{n_{k}} \rightarrow u_{0},\left\|u_{0}\right\|=1$, and $f\left(u_{0}\right)=0$. This contradicts $f$ is $(0, \varepsilon)$-epi on $B_{1}$. So $m(f)>0$ and $f$ is regular.

It is known that for a linear operator $f$, if $\lambda \in \sigma(f)$ and $|\lambda|>\alpha(f)$, then $\lambda$ is an eigenvalue of $f[15]$. We shall give an example later to show that for nonlinear operators, this property is not true. But if $f$ is positively homogeneous, we have the following result on eigenvalues in the spectrum. This theorem can be used to obtain existence results for some nonlinear operator equations as in the example which will be given later.

Theorem 4.5. Let $f: E \rightarrow E$ be a positively homogeneous operator and $\lambda \in \sigma(f)$ with $|\lambda|>\alpha(f)$. Then there exists $t_{0} \in(0,1]$ such that $\lambda / t_{0}$ is an eigenvalue of $f$.

Proof. $|\lambda|>\alpha(f)$ ensures that $\omega(\lambda I-f) \geq|\lambda|-\alpha(f)>0$ (see [9]). Let

$$
S=\{x \in E:\|x\|=1, \lambda x-t f(x)=0 \text { for some } t \in(0,1]\} .
$$

If $S=\emptyset$, then by the Homotopy property [17], $I-f / \lambda$ is $(0, r-\alpha(f) /|\lambda|)$-epi on $B_{1}$ for each $1>r>\alpha /|\lambda|$, since $f / \lambda$ is a $\alpha(f) /|\lambda|$-set contraction. It follows that $\lambda I-f$ is $(0, r|\lambda|-\alpha(f))$-epi on $B_{1}$. By Theorem 4.4, we know that $\lambda I-f$ is regular, so $\lambda \in \rho(f)$. This contradiction ensures that $S \neq \emptyset$. Thus there exists $t_{0} \in(0,1]$ and $x_{0} \in E$ with $\left\|x_{0}\right\|=1$, such that

$$
\lambda x_{0}-t_{0} f\left(x_{0}\right)=0,
$$

so $\lambda / t_{0}$ is an eigenvalue of $f$.

The following result, which generalizes a result of [15] (p. 85), shows that for odd and positively homogeneous mappings, the result on eigenvalues of linear operators remains valid. 
Theorem 4.6. Suppose $f: E \rightarrow E$ is odd and positively homogeneous, $\lambda \in \sigma(f)$ with $|\lambda|>\alpha(f)$. Then $\lambda$ is an eigenvalue of $f$.

Proof. Assume that $m(\lambda I-f)>0$. Then there exists $m>0$ such that

$$
\|(\lambda I-f) x\| \geq m\|x\| \text { for all } x \in E .
$$

Let $O_{1}=\{x:\|x\|<1\}$, since $f$ is odd, $\operatorname{deg}\left(I-f / \lambda, O_{1}, 0\right) \neq 0,[3]$. So for $k_{1}$ satisfying $0 \leq \alpha(f) /|\lambda|<k_{1}<1, I-f / \lambda$ is $\left(0, k_{1}-\alpha(f) /|\lambda|\right)$-epi $([17]$ Theorem 2.8). Hence, $\lambda I-f$ is $\left(0,|\lambda| k_{1}-\alpha(f)\right)$-epi on $B_{1}$. Also we know that $\omega(\lambda I-f) \geq|\lambda|-\alpha(f)>0$. Thus, $\lambda \in \rho(f)$. This contradiction shows that $m(\lambda I-f)=0$. Therefore there exists a sequence $\left\{x_{n}\right\}_{n=1}^{\infty} \in E$ such that

$$
\left\|\lambda x_{n}-f\left(x_{n}\right)\right\|<(1 / n)\left\|x_{n}\right\|
$$

Letting $u_{n}=x_{n} /\left\|x_{n}\right\|$, we have

$$
\left\|\lambda u_{n}-f\left(u_{n}\right)\right\|<1 / n \rightarrow 0, \quad \text { as } n \rightarrow \infty .
$$

Moreover, we have

$$
\omega(\lambda I-f) \alpha\left(\cup_{n=1}^{\infty} u_{n}\right) \leq \alpha\left(\cup_{n=1}^{\infty}(\lambda I-f) u_{n}\right)=0 .
$$

This implies $\alpha\left(\cup_{n=1}^{\infty} u_{n}\right)=0$. So $\left\{u_{n}\right\}$ has a convergent subsequence. Suppose that $u_{n_{k}} \rightarrow u_{0}$. Then $f\left(u_{0}\right)=\lambda u_{0}$ and $\left\|u_{0}\right\|=1$, so $\lambda$ is an eigenvalue of $f$.

The following result follows directly from Theorem 4.6, which generalizes the result in the spectral theory for linear compact operators.

Corollary 4.7. Suppose that $f$ is a compact, odd and homogeneous operator. Then for $\lambda \in \sigma(f)$, if $\lambda \neq 0, \lambda$ is an eigenvalue of $f$.

It is known that for a continuous linear operator $f$, the radius of the spectrum $r_{\sigma}(f)=\lim _{n \rightarrow \infty}\left\|f^{n}\right\|^{\frac{1}{n}}$. The following theorem gives an estimate for the radius of the spectrum of positively homogeneous maps.

Theorem 4.8. Let $E$ be a Banach space over $\mathbb{R}$ and $f: E \rightarrow E$ be a positively homogeneous operator with $\alpha(f)<\infty, \liminf _{n \rightarrow \infty}\left\|f^{n}\right\|^{\frac{1}{n}}<\infty$. If

$$
\lambda>\max \left\{\alpha(f), \liminf _{n \rightarrow \infty}\left\|f^{n}\right\|^{\frac{1}{n}}\right\},
$$

then $\lambda \in \rho(f)$. If also $\left\|x_{1}\right\|=\left\|x_{2}\right\|$ implies $\left\|f\left(x_{1}\right)\right\|=\left\|f\left(x_{2}\right)\right\|$, then

$$
r_{\sigma}(f) \leq \max \left\{\alpha(f), \liminf _{n \rightarrow \infty}\left\|f^{n}\right\|^{\frac{1}{n}}\right\} .
$$

Proof. Suppose that $\lambda>\max \left\{\alpha(f), \liminf _{n \rightarrow \infty}\left\|f^{n}\right\|^{\frac{1}{n}}\right\}$. Let

$$
V=\{x: \lambda x-t f(x)=0 \text { for some } t \in(0,1]\} .
$$

We claim that $V=\{0\}$. Indeed, otherwise assume $x_{0} \in V$ and $x_{0} \neq 0$. Let $t_{0} \in(0,1]$ be such that $\lambda x_{0}-t_{0} f\left(x_{0}\right)=0$. Then

$$
\|f\|=\sup _{\|x\|=1}\|f(x)\| \geq\left\|f\left(x_{0} /\left\|x_{0}\right\|\right)\right\| \geq \lambda .
$$


Also

$$
\left\|f^{2}\left(x_{0} /\left\|x_{0}\right\|\right)\right\|=\left\|f\left(\left(\lambda x_{0}\right) /\left(t_{0}\left\|x_{0}\right\|\right)\right)\right\| \geq \lambda^{2} / t_{0}^{2} .
$$

So we have $\left\|f^{2}\right\| \geq \lambda^{2}$. By induction, we obtain that $\left\|f^{n}\right\|^{\frac{1}{n}} \geq \lambda$. This contradicts $\lambda>\liminf _{n \rightarrow \infty}\left\|f^{n}\right\|^{\frac{1}{n}}$. Now, $\lambda I-f=\lambda(I-f / \lambda)$ and $f / \lambda$ is an $\alpha(f) /|\lambda|$-set contraction. So, by the homotopy property [17], $I-f / \lambda$ is $(0, r-\alpha(f) /|\lambda|)$-epi on $B_{1}$ for every $r$ satisfying $\alpha(f) /|\lambda|<r<1$. Thus $\lambda I-f$ is $(0, r|\lambda|-\alpha(f))$-epi on $B_{1}$. Furthermore, $\omega(\lambda I-f)>0$. Theorem 4.4 implies that $\lambda \in \rho(f)$.

In the case that $\left\|x_{1}\right\|=\left\|x_{2}\right\|$ implies $\left\|f\left(x_{1}\right)\right\|=\left\|f\left(x_{2}\right)\right\|,(4.2)$ is also true for $\lambda<0$. So by the same proof as above, we obtain that if

$$
|\lambda|>\max \left\{\alpha(f), \liminf _{n \rightarrow \infty}\left\|f^{n}\right\|^{\frac{1}{n}}\right\},
$$

then $\lambda \in \rho(f)$. Therefore we have (4.1).

The following example shows that the estimate in Theorem 4.8 is best possible.

Example 4.9. Let $f(x)=a\|x\| e$, then $f$ is positively homogeneous and even. $a$ is an eigenvalue of $f$ and $\left\|f^{n}\right\|=a^{n}$. Hence liminf $\left\|f^{n}\right\|^{\frac{1}{n}}=a$ and $r_{\sigma}(f)=a$.

\section{Comparison AND NONEMPtiness OF SPECTRA}

We begin this section with an example concerning eigenvalues and the spectrum $\sigma_{f m v}(f)$.

Example 5.1. Let $f: \mathbb{R} \rightarrow \mathbb{R}$ be the function $f(x)=x^{3}$. Then $\sigma_{f m v}(f)=\emptyset$ (see [9]). We will show that $\sigma(f)=\{0\} \cup\{$ eigenvalues of $f\}$. In fact, for every $\lambda \in(0, \infty)$, we have $f\left(\lambda^{\frac{1}{2}}\right)=\lambda \lambda^{\frac{1}{2}}$. Thus $\lambda$ is an eigenvalue of $f$, so $(0, \infty) \subset \sigma(f)$. Next, $0 \in \sigma(f)$ since $m(f)=0$. Furthermore, let $\lambda \in(-\infty, 0)$, then $|\lambda x-f(x)|=\left|\lambda x-x^{3}\right| \geq-\lambda|x|$ for $x \in \mathbb{R}$. Hence $m(\lambda I-f) \geq-\lambda>0$. Also, $\omega(\lambda I-f)>0$ and $(\lambda I-f) x=\lambda x-x^{3}$ is $(0, \varepsilon)$-epi for all $\varepsilon>0$. This implies that $\nu(\lambda I-f)>0$. Therefore, $(-\infty, 0)=\rho(f),[0, \infty)=\sigma(f)$ and $\sigma(f)=\{0\} \cup\{$ eigenvalues of $f\}$.

The following is an interesting result of the theory.

Theorem 5.2. Suppose $f: E \rightarrow E$ is continuous and $f(0)=0$. Then we have

$$
\sigma_{l i p}(f) \supseteq \sigma(f) \supseteq \sigma_{f m v}(f) .
$$

Proof. We will prove that $\rho_{\text {lip }}(f) \subseteq \rho(f) \subseteq \rho_{f m v}(f)$.

(a) Assume that $\lambda \in \rho(f)$. Then $\omega(\lambda I-f)>0$ and $m(\lambda I-f)>0$. Hence there exists $m>0$ such that

$$
\|(\lambda I-f)(x)\| \geq m\|x\| \text { for all } x \in E .
$$

This ensures that

$$
d(\lambda I-f)=\liminf _{\|x\| \rightarrow \infty} \frac{\|(\lambda I-f)(x)\|}{\|x\|} \geq m>0 .
$$


Moreover, $\nu(\lambda I-f)>0$ implies that there exists $\varepsilon>0$ such that $\lambda I-f$ is $(0, \varepsilon)$-epi on $B_{r}$ with $r>0$. So $\lambda I-f$ is stably-solvable [9]. Thus $\lambda \in \rho_{f m v}(f)$ and therefore $\rho(f) \subseteq \rho_{f m v}(f)$.

(b) Suppose that $\lambda \in \rho_{\text {lip }}(f)$, then $\lambda I-f$ is one to one, onto, and $(\lambda I-f)^{-1}$ is a Lipschitz map [15]. Let $L>0$ be the Lipschitz constant. Then

$$
\left\|(\lambda I-f) x_{1}-(\lambda I-f) x_{2}\right\| \geq(1 / L)\left\|x_{1}-x_{2}\right\| \text { for all } x_{1}, x_{2} \in E .
$$

Letting $x_{2}=0$, we have $\left\|(\lambda I-f) x_{1}\right\| \geq(1 / L)\left\|x_{1}\right\|$ for all $x_{1} \in E$. Hence $m(\lambda I-f)>0$. Also, by $(5.1), \omega(\lambda I-f) \geq 1 / L>0$.

Let $r>0$ and $O_{r}=\{x:\|x\|<r\} . \lambda I-f: O_{r} \rightarrow E$ is continuous, injective and $(1 / L)$-proper [17]. Furthermore $(\lambda I-f) O_{r}$ is open because $(\lambda I-f)^{-1}$ is continuous. By our assumption $(\lambda I-f)(0)=0$. By Theorem 2.3 of [17], $\lambda I-f$ is $(0, k)$-epi on $B_{r}$ for each nonnegative $k$ satisfying the condition $k<L$. Hence $\nu(\lambda I-f)>0, \lambda \in \rho(f)$ and $\rho_{\text {lip }}(f) \subseteq \rho(f)$.

The following example shows that $\left.\sigma_{\text {lip }}(f) \supsetneqq \sigma_{(} f\right)$.

Example 5.3. Let $\psi: \mathbb{R} \rightarrow \mathbb{R}$ be defined by

$$
\psi(x)= \begin{cases}x & \text { for } \quad x \leq 1 \\ 1 & \text { for } 1<x<2 \\ x-1 & \text { for } x \geq 2\end{cases}
$$

Let $f=I-\psi$. Then for $x \in \mathbb{R}$ we have $(1 / 2)\|x\| \leq\|\psi(x)\| \leq 2\|x\|$ and so $\|f(x)\| \leq 3\|x\|$. Also, $\omega(I-f)>0$ for $f$ is a compact map. $I-f$ is $(0, \varepsilon)$-epi for all $\varepsilon>0$ on every $[-n, n]$. Hence $\lambda=1 \in \rho(f)$.

Obviously, $\lambda I-f=\psi$ is not one to one, so $1 \in \sigma_{\text {lip }}(f)$. Thus $\sigma(f) \varsubsetneqq$ $\sigma_{\text {lip }}(f)$.

Example 5.1 shows that $\sigma(f) \supsetneqq \sigma_{f m v}(f)$.

The following two results discuss the spectrum for positively homogeneous maps and maps that are derivable at 0 respectively.

Theorem 5.4. Suppose $f$ is a positively homogeneous map and $\lambda \in \sigma(f) \backslash$ $\sigma_{f m v}(f)$. Then one of the following cases occur:

1. $\lambda I-f$ is not injective;

2. $(\lambda I-f)^{-1}$ is not continuous.

Proof. Suppose that $\lambda \in \sigma(f) \backslash \sigma_{f m v}(f)$ and $\lambda I-f$ is injective. We shall show that $(\lambda I-f)^{-1}$ is not continuous. Firstly $\lambda \notin \sigma_{f m v}(f)$ ensures that $\omega(\lambda I-f)>0$ and $\lambda I-f$ is surjective. Also $\lambda I-f$ is injective implies that $\lambda x-f(x) \neq 0$ for each $x \neq 0$. Hence $\lambda I-f$ is $\omega(\lambda I-f)$-proper [17]. Assume $(\lambda I-f)^{-1}$ is continuous, then $\lambda I-f$ maps every open ball $O_{r}$ to an open set. It follows that $\lambda I-f$ is $(0, k)$-epi for each nonnegative $k$ satisfying $k<1 / \omega(\lambda I-f)$. By Theorem 4.4, we have $\lambda \in \sigma(f)$. This contradiction shows that $(\lambda I-f)^{-1}$ is not continuous.

Theorem 5.5. Let $f: E \rightarrow E$ be derivable at 0 with derivative $T$ and $\lambda \in \sigma(f) \backslash \sigma_{f m v}(f)$. Then one of the following cases occur:

1. $\lambda$ is an eigenvalue of $f$; 
2. $\lambda I-f$ is not injective;

3. $(\lambda I-f)^{-1}$ is not continuous;

4. $\lambda \in \sigma(T)$.

Proof. Let $\lambda \in \sigma(f) \backslash \sigma_{f m v}(f)$, then $\lambda I-f$ is onto and $\omega(\lambda I-f)>0$. Now suppose that $m(\lambda I-f)=0$. It follows that for each $n \in \mathbb{N}$, there exists $x_{n} \in E$ satisfying

$$
\left\|\lambda x_{n}-f\left(x_{n}\right)\right\|<(1 / n)\left\|x_{n}\right\| .
$$

Assume there exists a subsequence $\left\{x_{n_{k}}\right\}_{k=1}^{\infty}$ of $\left\{x_{n}\right\}_{n=1}^{\infty}$ with $\left\|x_{n_{k}}\right\| \rightarrow \infty$ as $k \rightarrow \infty$. Then

$$
d(\lambda I-f)=\liminf _{\|x\| \rightarrow \infty} \frac{\|\lambda x-f(x)\|}{\|x\|}=0 .
$$

This contradicts $\lambda \in \rho(f)$. So, $\left\{\left\|x_{n}\right\|\right\}_{n=1}^{\infty}$ is bounded and

$$
\omega(\lambda I-f) \alpha\left(\cup_{n=1}^{\infty} x_{n}\right) \leq \alpha\left(\cup_{n=1}^{\infty}(\lambda I-f) x_{n}\right)=0 .
$$

This implies $\left\{x_{n}\right\}_{n=1}^{\infty}$ has a convergent subsequence. Suppose $x_{n} \rightarrow x_{0}$ as $n \rightarrow \infty$. If $x_{0} \neq 0, \lambda$ is an eigenvalue of $f$. In the case $x_{0}=0$, we have

$$
\left\|\lambda x_{n}-T x_{n}-R x_{n}\right\| /\left\|x_{n}\right\|<1 / n \rightarrow 0 .
$$

Thus $\lambda \in \sigma(T)$ since $\left\|R x_{n}\right\| /\left\|x_{n}\right\| \rightarrow 0$.

In the case $m(\lambda I-f)>0$, assume that $\lambda I-f$ is injective, by the same argument as that in the proof of Theorem $5.4,(\lambda I-f)^{-1}$ is not continuous.

A well known result in the spectral theory for linear operators is that the spectrum of a continuous linear operator, which is defined on a complex Banach space, is not empty. In the nonlinear case, for the spectrum $\sigma_{f m v}(f)$, this property does not hold (see the counterexample in [9]). An open question in $[15]$ is the following:

Question 5.1. Suppose that $E$ is a Banach space over the complex field $\mathbb{C}$ and that $A \in \operatorname{Lip}(E)$. Is the spectrum of $A, \sigma_{\text {lip }}(f)$, nonempty?

In the following, an operator $f$ is given which satisfies $\sigma(f)=\sigma_{\text {lip }}(f)=$ $\sigma_{f m v}(f)=\emptyset$.

Example 5.6. Let $f: \mathbb{C}^{2} \rightarrow \mathbb{C}^{2}$ be defined by

$$
f(x, y)=(\bar{y}, i \bar{x}), \quad(x, y) \in \mathbb{C}^{2} .
$$

Then $f$ is a continuous map and $f \in \operatorname{Lip}\left(\mathbb{C}^{2}\right)$. For $\lambda \in \mathbb{C}$ and $\lambda \neq 0$, we will show that $\lambda I-f: \mathbb{C}^{2} \rightarrow \mathbb{C}^{2}$ is one to one, onto and $(\lambda I-f)^{-1} \in \operatorname{Lip}\left(\mathbb{C}^{2}\right)$. Firstly, suppose that $(\lambda I-f)\left(x_{1}, y_{1}\right)=(\lambda I-f)\left(x_{2}, y_{2}\right)$, then

$$
|\lambda|^{2}\left(x_{1}-x_{2}\right)=\bar{\lambda}\left(\overline{y_{1}}-\overline{y_{2}}\right) \text { and } i\left(x_{2}-x_{1}\right)=\bar{\lambda}\left(\overline{y_{1}}-\overline{y_{2}}\right) \text {. }
$$

Hence $x_{1}=x_{2}, y_{1}=y_{2}$. Secondly, for $(x, y) \in \mathbb{C}^{2}$, let

$$
u=\frac{\bar{\lambda} x+\bar{y}}{|\lambda|^{2}+i}, \quad v=\frac{i \bar{\lambda} y-\bar{x}}{|\lambda|^{2} i+1} .
$$


Then $(\lambda I-f)(u, v)=(x, y)$. Hence $\lambda I-f$ is onto. Next, let $g=(\lambda I-f)^{-1}$, and for $\left(x_{1}, y_{1}\right),\left(x_{2}, y_{2}\right) \in \mathbb{C}^{2}$, let $x=x_{1}-x_{2}, y=y_{1}-y_{2}$. Then

$$
\left|g\left(x_{1}, y_{1}\right)-g\left(x_{2}, y_{2}\right)\right|^{2}=\left|\frac{1}{|\lambda|^{2}+i}\right|^{2}|\bar{\lambda} x+\bar{y}|^{2}+\left|\frac{1}{i-|\lambda|^{2}}\right|^{2}|i \bar{x}+\bar{\lambda} y|^{2} .
$$

Letting $r=\left|\frac{1}{|\lambda|^{2}+i}\right|>0$, we have

$$
\left|g\left(x_{1}, y_{1}\right)-g\left(x_{2}, y_{2}\right)\right|^{2} \leq r^{2}(|\lambda|+1)^{2}\left(|x|^{2}+|y|^{2}\right) .
$$

So, $g$ is a Lipschitz map with constant $r(|\lambda|+1)$.

In the case $\lambda=0,|f(x)|=|x|$. Also, $f$ is one to one, onto with $\left|f^{-1}(x)\right|=$ $|x|$. Hence, $\rho_{\text {lip }}(f)=\mathbb{C}$ and $\sigma_{\text {lip }}(f)=\emptyset$. By Theorem 5.2, $\sigma(f)=\sigma_{\text {lip }}(f)=$ $\sigma_{f m v}(f)=\emptyset$.

Remark 5.7. In [16], the authors showed that $\sigma_{\text {lip }}(f)$ is always nonempty in the one-dimensional case and asked whether this is also true in higher dimensions. In [1] (which was seen after this part of the work had been completed), the authors gave a negative answer to this question by using Example 5.6. We found Example 5.6 in [13] where it was used to show another fact.

We close this section with the following result regarding operators which are asymptotically linear or derivable at 0 .

Proposition 5.8. Let $f: E \rightarrow E$ be continuous and $f=T+R$, where $T$ is a linear operator and $R$ satisfies one of the following conditions:

1. $\frac{\|R(x)\|}{\|x\|} \rightarrow 0$ as $\|x\| \rightarrow 0$.

2. $\frac{\|R(x)\|}{\|x\|} \rightarrow 0$ as $\|x\| \rightarrow \infty$.

Then $\lambda \in \sigma(f)$ provided $\lambda$ is an eigenvalue of $T$.

Proof. Let $x_{0} \in E$ and $x_{0} \neq 0$ be such that $T\left(x_{0}\right)=\lambda x_{0}$. For $r \in \mathbb{R}$ with $r \geq 0$ we have

$$
\left\|\lambda r x_{0}-f\left(r x_{0}\right)\right\|=\left\|R\left(r x_{0}\right)\right\| .
$$

So, in case (1), letting $r \rightarrow 0$ and in case (2) letting $r \rightarrow \infty$, we have

$$
\frac{\left\|\lambda r x_{0}-f\left(r x_{0}\right)\right\|}{r\left\|x_{0}\right\|} \rightarrow 0 .
$$

This implies that $m(\lambda I-f)=0$. Hence $\lambda \in \sigma(f)$.

\section{Applichtions}

In this section, firstly by applying the theory, we shall study the solvability of a global Cauchy problem following the work of [14], we shall obtain the existence result by different method. Then two well known theorems will be generalized by using the theory.

We shall use the classical space $C[0,1]$ with the norm

$$
\|x\|=\max _{t \in[0,1]}|x(t)| \text {. }
$$


We recall that a cone $K$ in a Banach space $E$ is a closed subset of $E$ such that

(1) $x, y \in K, a, b \geq 0$ imply $a x+b y \in K$;

(2) $x \in K$ and $-x \in K$ imply $x=0$.

A cone $K$ is said to be normal if there exists a constant $\gamma>0$ such that

$$
\|x+y\| \geq \gamma\|x\|
$$

for every $x, y \in K$.

Example 6.1. We look for a non-trivial solution of the following global Cauchy problem depending on a parameter

$$
x^{\prime}(t)=\lambda \sqrt{x^{2}(t)+x^{2}(1-t)}, \quad x(0)=0, t \in[0,1] .
$$

Changing the problem into an integral equation we study the existence of an eigenvalue and eigenvector of the operator

$$
F x(t)=\int_{0}^{t} \sqrt{x^{2}(s)+x^{2}(1-s)} d s .
$$

It is easily verified that $F: C[0,1] \rightarrow C[0,1]$ is positively homogeneous, order preserving, and $\|F x\| \leq \sqrt{2}\|x\|$.

Now we shall prove that $\mu I-F$ is not surjective for every $0<\mu<1 / \sqrt{2}$ and so $[0,1 / \sqrt{2}] \subset \sigma(T)$. Assume it is surjective, then there exists $x_{0} \in$ $C[0,1]$ such that $\mu x_{0}-T x_{0}=\mu$. For every $t \in[0,1]$,

$$
\mu\left(x_{0}(t)-1\right)=F x_{0}(t) \geq 0 \Longrightarrow x_{0}(t) \geq 1 .
$$

So for each natural number $n, F^{n} x_{0} \geq F^{n} 1$. On the other hand,

$$
F x_{0} \leq \mu x_{0} \text {, so } F^{n} x_{0} \leq \mu^{n} x_{0} .
$$

Hence

$$
\mu^{n} x_{0} \geq F^{n} x_{0} \geq F^{n}(1)
$$

This implies that

$$
(\sqrt{2})^{n} \mu^{n} x_{0} \geq(\sqrt{2})^{n} F^{n}(1) \geq 0 .
$$

Also we know that $K=\{x(t) \in C[0,1]: x(t) \geq 0\}$ is a normal cone in $C[0,1]$ and $(\sqrt{2})^{n} \mu^{n} \rightarrow 0$ as $n \rightarrow \infty$. Thus we obtain that $(\sqrt{2})^{n} F^{n}(1) \rightarrow$ $0(n \rightarrow \infty)$. This contradicts $F^{n}(1) \geq(1 / \sqrt{2})^{n} t$. So, $\mu I-F$ is not onto.

Assume that for some $-1 / \sqrt{2}<\mu<0, \mu I-F$ is onto. Then for each $y \in$ $C[0,1]$, there exists $x \in C[0,1]$, such that $\mu x-F x=y$. Hence $(-\mu)(-x)-$ $F(-x)=y$. So, $-\mu-F$ is onto. This is a contradiction since $1 / \sqrt{2}>-\mu>0$.

By the above argument, $[-1 / \sqrt{2}, 1 / \sqrt{2}] \subset \sigma(F)$ since the spectrum of $F$ is closed. Also, we know that $F$ is a compact map [14], so $\alpha(F)=0$. By Theorem 4.5 , there exist $\mu_{1} \geq 1 / \sqrt{2}$ and $\mu_{2} \leq-1 / \sqrt{2}$ such that $\mu_{1}$ and $\mu_{2}$ are eigenvalues of $F$. Moreover, by Theorem 4.8, we obtain that $1 / \sqrt{2} \leq\left|\mu_{i}\right| \leq \liminf _{n \rightarrow \infty}\left\|F^{n}\right\|^{1 / n}$. Therefore, problem (6.1) has at least two non-trivial solutions. 
Remark 6.2. It was known that for the above operator $F,(1 / \sqrt{2}) \ln (1+$ $\sqrt{2}$ ) is the only positive eigenvalue of $F$ (see [14]). So, for each $\lambda \in[-1 / \sqrt{2}$, $1 / \sqrt{2}], \lambda$ is not an eigenvalue of $F$. This shows that Theorem 4.6 is not true for positively homogeneous maps. It is well known that it is true for linear operators.

The following theorem is a generalization of the well known Birkoff-Kellogg theorem.

Theorem 6.3. Let $E$ be an infinite dimensional Banach space and $S$ be the unit sphere of $E$. Let $f: S \rightarrow E$ be continuous and bounded. Assume that $\inf _{x \in S}\|f(x)\|>\alpha(f)$. Then for every complex number $\lambda$ with $\lambda \neq 0$ there exists $r>0$ such that $r \lambda$ is an eigenvalue of $f$. In particular, $f$ has a positive and a negative eigenvalue.

Proof. Let $\tilde{f}: E \rightarrow E$ be the positively homogeneous operator which is defined as follows:

$$
\tilde{f}(x)= \begin{cases}\|x\| f(x /\|x\|) & \text { if } x \neq 0 \\ 0 & \text { if } x=0\end{cases}
$$

Then we have

$$
d(\tilde{f})=\liminf _{\|x\| \rightarrow \infty} \frac{\|\tilde{f}(x)\|}{\|x\|}=\inf _{x \in S}\|f(x)\|, \quad|\tilde{f}|=\limsup _{\|x\| \rightarrow \infty} \frac{\|\tilde{f}(x)\|}{\|x\|}=\sup _{x \in S}\|f(x)\|,
$$

and $\alpha(\tilde{f})=\alpha(f)$ (see [14]). Let $B(\tilde{f})$ be the set of all asymptotic bifurcation points of $\tilde{f}$. By Theorem 11.1.1 of [9], there exists $\mu>0$ such that $\mu \in B(\tilde{f})$. Let $\left\{\mu_{n}\right\}_{n=1}^{\infty}$ and $\left\{x_{n}\right\}_{n=1}^{\infty} \in E$ be sequences such that $\mu_{n} \rightarrow \mu,\left\|x_{n}\right\| \rightarrow \infty$ as $n \rightarrow \infty$ and $\tilde{f}\left(x_{n}\right)=\mu_{n} x_{n}$. Then we obtain

$$
\frac{\left\|(\mu I-\tilde{f}) x_{n}\right\|}{\left\|x_{n}\right\|} \rightarrow 0, \quad \text { as } n \rightarrow \infty .
$$

This implies that $d(\mu I-\bar{f})=0$. So, by Theorem $5.2, \mu \in \sigma_{f m v}(\tilde{f}) \subset \sigma(\tilde{f})$. Assume that $\mu \leq \alpha(\tilde{f})$, by our assumption $\alpha(\tilde{f})=\alpha(f)<d(\tilde{f})$, so $\mu<d(\tilde{f})$. Hence

$$
d(\mu I-\tilde{f}) \geq d(\tilde{f})-\mu>0, \quad(\text { see }[9]) .
$$

This contradicts $d(\mu I-\tilde{f})=0$. Thus we have $\mu>\alpha(\tilde{f})$. By Theorem 4.5, there exists $t_{0} \in(0,1]$ such that $\mu / t_{0}$ is an eigenvalue of $\tilde{f}$. Let $x_{0} \in E$ with $\left\|x_{0}\right\|=1$ be such that $\tilde{f}\left(x_{0}\right)=\left(\mu / t_{0}\right) x_{0}$. Then $f\left(x_{0}\right)=r x_{0}$, where $r=\mu / t_{0}$.

For every complex number $\lambda$ with $|\lambda|=1$, writing $\bar{\lambda}=e^{i \theta}$, we have

$$
\inf _{x \in S}\|\bar{\lambda} f(x)\|=\inf _{x \in S}\|f(x)\|>\alpha(f)=\alpha(\bar{\lambda} f) .
$$

By the above argument, there exists $r>\alpha(f)$ and $x_{0} \in E$ with $x_{0} \neq 0$ such that $\bar{\lambda} f\left(x_{0}\right)=r x_{0}$. Thus $f\left(x_{0}\right)=(r \lambda) x_{0}$. In the case $|\lambda| \neq 1$, let $\lambda_{1}=\lambda /|\lambda|$. By the same argument, there exists $r>0$ such that $r \lambda$ is an eigenvalue of $f$. 
Remark 6.4. The Birkoff-Kellogg theorem which was proved in [9] (Theorem 10.1.5) is the special case of Theorem 6.3 when $f$ is compact, and they showed only that $f$ has a positive eigenvalue.

The following example shows that there exists a map $f$ to which Theorem 10.1.5 of [9] does not apply but Theorem 6.3 can be used.

Example 6.5. Let $B_{1}=\{x \in E:\|x\| \leq 1\}$ and $g: E \rightarrow B_{1}$ be the radial retraction of $E$ onto the unit ball, that is

$$
g(x)= \begin{cases}x /\|x\| & \text { if }\|x\|>1 \\ x & \text { if } 0 \leq\|x\| \leq 1\end{cases}
$$

Since $g(A) \subset \operatorname{co}(A \cup 0), g$ is a 1 -set contraction [3]. Let $y \in E$ with $\|y\|>2$ and $f: S \rightarrow E$ be defined by

$$
f(x)=y+g(x), x \in E .
$$

Then,

$$
\inf _{x \in S}\|f(x)\|=\inf _{x \in S}\|y+g(x)\| \geq\|y\|-\sup _{x \in S}\|g(x)\|=\|y\|-1>1 .
$$

Next,

$$
\alpha(f)=\alpha(y+g)=\alpha(g)=1 .
$$

So, $\inf _{x \in S}\|f(x)\|>\alpha(f)$. Furthermore, we have

$$
\sup _{\|x\|=1}\|f(x)\|=\sup _{\|x\|=1}\|y+g(x)\| \leq\|y\|+1 .
$$

Hence, $f$ satisfies the conditions of Theorem 6 .3. So, for every $z \in \mathbb{C}$, there exists $\lambda>1$ such that $\lambda z$ is an eigenvalue of $f$.

Theorem 6.3 enables us to give the following generalization of Theorem 10.1.2 of [9], who considered compact maps.

Theorem 6.6. Let $S$ be the unit sphere in an infinite dimensional Banach space $E$ and let $f: S \rightarrow S$ be a continuous strict set contraction. Then every $\lambda \in \mathbb{K},|\lambda|=1$, is an eigenvalue of $f$. In particular, $f$ has a fixed point and an antipodal point.

Proof. Since $\alpha(f)<1$, we have

$$
\inf _{x \in S}\|f(x)\|=1>\alpha(f), \quad \sup _{x \in S}\|f(S)\|<+\infty .
$$

By Theorem 6.3, for every complex number $|\lambda|=1$, there exists $\alpha>0$, such that $\alpha \lambda$ is an eigenvalue of $f$. Thus there exists $x_{\lambda} \in S$ such that $f\left(x_{\lambda}\right)=\alpha \lambda x_{\lambda}$. Since $\left\|f\left(x_{\lambda}\right)\right\|=1,|\alpha \lambda|=\alpha=1$, so $\lambda$ is an eigenvalue of $f$.

In particular, for $\lambda=1$, there exists $x \in S$, such that $f(x)=x$. For $\lambda=-1$, there exists $x \in S$, such that $f(x)=-x$.

Our theory also enables us to give a generalization of the Hopf theorem on spheres, Theorem 10.1.6 of [9]. Firstly, we need the following lemma. We 
shall let $E^{*}$ denote the dual space of $E$ and $J: E \rightarrow E^{*}$ the duality mapping, that is, for $x \in E$,

$$
J(x)=\left\{x^{*} \in E^{*}: x^{*}(x)=\|x\|^{2}=\left\|x^{*}\right\|^{2}\right\} .
$$

We recall that for a linear operator $A$ in a Hilbert space $E$, the numerical range of $A$ is the set of values of $(A x, x)$ for all $x$ with $\|x\|=1$. It is known that the numerical range of $A$ is a convex set. Let $V(A)$ be the numerical range of $A$, then $\sigma(A) \subset \overline{V(A)}$.

For a nonlinear operator $f$, we have the following.

Lemma 6.7. Suppose $f: E \rightarrow E$ is a continuous operator. Let

$$
V(f)=\left\{\frac{\left(f(x), x^{*}\right)}{\|x\|^{2}}\right\} \cup\{0\}, \quad x \in E, x^{*} \in J(x) .
$$

Then $\lambda \in \overline{\mathrm{co}} V(f)$ provided that $\lambda \in \sigma(f)$ and $|\lambda|>\alpha(f)$, where $\overline{\mathrm{co}}$ denotes the closed convex hull.

Proof. We shall prove that $|\lambda|>\alpha(f)$ and $\operatorname{dist}(\lambda, \overline{\operatorname{co}} V(f))>0$ implies that $\lambda \in \rho(f)$.

If $|\lambda|>\alpha(f)$ and $\operatorname{dist}(\lambda, \overline{\operatorname{co}} V(f))>0$, then

$$
\begin{aligned}
0<d & =\operatorname{dist}(\lambda, \operatorname{conv} V(f)) \\
& \leq\left|\lambda-\frac{\left(f(x), x^{*}\right)}{\|x\|^{2}}\right| \\
& =\left|\frac{\lambda\left(x, x^{*}\right)-\left(f(x), x^{*}\right)}{\|x\|^{2}}\right| \\
& =\frac{\left|\left(\lambda x-f(x), x^{*}\right)\right|}{\|x\|^{2}} \\
& \leq \frac{\|\lambda x-f(x)\|}{\|x\|} .
\end{aligned}
$$

So, $\|\lambda x-f(x)\| \geq d\|x\|$. This implies that $m(\lambda I-f)>0$. Also, $\omega(\lambda I-$ $f(x))>0$ since $|\lambda|>\alpha(f)$. Let

$$
M=\{x \in E: \lambda x-t f(x)=0, t \in[0,1]\} .
$$

For $x \in M$, suppose $\lambda x=t f(x)$ with $t \neq 0$. Then

$$
\left(f(x), x^{*}\right) /\|x\|^{2}=\left((\lambda / t) x, x^{*}\right) /\|x\|^{2}=\lambda / t .
$$

Hence,

$$
\lambda=t\left(f(x), x^{*}\right) /\|x\|^{2} \in \overline{\mathrm{co}} V(f) .
$$

This contradicts our assumption $d=\operatorname{dist}(\lambda, \overline{\operatorname{co}} V(f))>0$. Thus, $M=\{0\}$. By the homotopy property [17], $\lambda I-f$ is $(0, \varepsilon)$-epi for some $\varepsilon>0$, thus $\nu(\lambda I-f)>0$, and so $\lambda I-f$ is regular. This shows that

$$
\{\lambda:|\lambda|>\alpha(f)\} \cap \sigma(f) \subset \overline{\operatorname{co}} V(f) .
$$

Let $S^{n}=\left\{x \in \mathbb{R}^{n+1}:\|x\|=1\right\}$. The following theorem is a generalization of Theorem 10.1 .6 of [9]. 
Theorem 6.8. 1 . Let $f: S^{n} \rightarrow \mathbb{R}^{n+1}$ be continuous. Assume $\langle f(x), x\rangle=$ 0 for all $x \in S^{n}$, where $\langle\cdot, \cdot\rangle$ is the Euclidean inner product on $\mathbb{R}^{n+1}$. Then, $f$ vanishes at some point $x \in S^{n}$ provided either $n$ is an even number or $f\left(S^{n}\right)$ is contained in a proper subspace of $\mathbb{R}^{n+1}$.

2. Let $E$ be an infinite dimensional Banach space and $f: S \rightarrow E$ be a continuous compact mapping. Assume that $\left(f(x), x^{*}\right)=0$ for all $x^{*} \in J(x), x \in S$. Then $\inf _{x \in S}\|f(x)\|=0$.

Proof. (1) Let

$$
\tilde{f}(x)= \begin{cases}\|x\| f(x /\|x\|) & \text { if } x \neq 0, \\ 0 & \text { if } x=0 .\end{cases}
$$

Then for every $x \in \mathbb{R}^{n+1}$ with $x \neq 0$,

$$
\langle\tilde{f}(x), x\rangle=\|x\|\langle f(x /\|x\|), x /\|x\|\rangle=0 .
$$

So, $V(\tilde{f})=\{0\}$. Also, $\alpha(\tilde{f})=0$ since $\mathbb{R}^{n+1}$ is finite dimensional. By Lemma 6.7 , we have $\sigma(\tilde{f}) \subset\{0\}$.

(a) Assume that $n$ is even. Let $B(\tilde{f})$ denote the set of all asymptotic bifurcation points of $\tilde{f}$, then by Theorem 11.1 .3 of $[9], B(\tilde{f}) \neq \emptyset$. By Proposition 3.11, $B(\tilde{f}) \subset \sigma(\tilde{f})$. Hence, $0 \in B(\tilde{f})$. Suppose that $\left\{x_{n}\right\}_{n=1}^{\infty} \in \mathbb{R}^{n+1}$ and $\lambda_{n} \in \mathbb{K}$ satisfy $\left\|x_{n}\right\| \rightarrow \infty, \lambda_{n} \rightarrow 0$ as $n \rightarrow \infty$ and $\lambda_{n} x_{n}=\tilde{f}\left(x_{n}\right)$. Then

$$
\tilde{f}\left(x_{n} /\left\|x_{n}\right\|\right)=\lambda_{n} x_{n} /\left\|x_{n}\right\| \rightarrow 0 .
$$

Moreover, $\left\{x_{n} /\left\|x_{n}\right\|\right\}_{n=1}^{\infty}$ has a convergent subsequence $x_{n_{k}} /\left\|x_{n_{k}}\right\| \rightarrow x_{0}(k \rightarrow$ $\infty)$. Thus $\left\|x_{0}\right\|=1$ and $\tilde{f}\left(x_{0}\right)=f\left(x_{0}\right)=0$.

(b) In the case that $f\left(S^{n}\right)$ is contained in a proper subspace of $\mathbb{R}^{n+1}$, $\tilde{f}: \mathbb{R}^{n+1} \rightarrow \mathbb{R}^{n+1}$ can not be surjective. So $0 \in \sigma(\tilde{f})$. Also, we know that $\omega(\tilde{f})=\infty$ since $\mathbb{R}^{n+1}$ is finite dimensional. Firstly, if $d(\tilde{f})=0$, it follows that there exists $\left\{x_{n}\right\}_{n=1}^{\infty} \in \mathbb{R}^{n+1}$ with $\left\|x_{n}\right\|=1$ such that

$$
\tilde{f}\left(x_{n}\right)=f\left(x_{n}\right) \rightarrow 0(n \rightarrow \infty) .
$$

So, $f\left(x_{0}\right)=0$ for some $x_{0} \in \mathbb{R}^{n+1}$ and $\left\|x_{0}\right\|=1$. Secondly, if $d(\tilde{f})>0$, then $0 \in \mathbb{K} \backslash \sigma_{\pi}(\tilde{f})$ and $1 \notin \sigma(\tilde{f})$. By Theorem 11.1.2 of [9], we have $B(\tilde{f}) \cup \sigma_{\omega}(\tilde{f})$ separates 1 from 0 . So, $B(\tilde{f}) \cup \sigma_{\omega}(\tilde{f}) \neq \emptyset$. This implies that $B(\tilde{f}) \neq \emptyset$ since $\sigma_{\omega}(\tilde{f})=\emptyset$. By the same argument with that in (a), there exists $x_{0} \in S^{n}$ such that $f\left(x_{0}\right)=0$.

(2) Suppose $E$ is an infinite Banach space. Let $\tilde{f}$ be as in (1), then $V(\tilde{f})=\{0\}$. By Theorem 8.2.1 of [9], $\Sigma(\tilde{f}) \neq \emptyset$ (according to [9], $\Sigma(\tilde{f})=$ $\{\lambda \in \mathbb{K}: d(\lambda I-\tilde{f})=0\})$. Again applying Lemma 6.7, we have

$$
\Sigma(\tilde{f}) \subset \sigma(\tilde{f}) \subset V(\tilde{f}) .
$$

So, $0 \in \Sigma(\tilde{f})$. Thus there exist $x_{n} \in E$ with $\left\|x_{n}\right\|=1$ such that $\tilde{f}\left(x_{n}\right)=$ $f\left(x_{n}\right) \rightarrow 0$. Hence $\inf _{x \in S}\|f(x)\|=0$.

Remark 6.9. Theorem 10.1.6 of [9] only proved the case 1 of Theorem 6.8 and $n$ is even. 
Acknowledgment. The author wishes to thank Professor J. R. L. Webb for valuable discussions.

\section{REFERENCES}

[1] J. Appell and M. Dörfner, Some spectral theory for nonlinear operators, Nonlinear Anal. 28 (1997), 1955-1976.

[2] S. R. Bhatt and S. J. Bhatt, On the nonlinear spectrum of Furi and Vignoli, Math. Today, 2 (1984), 49-64.

[3] K. Deimling, Nonlinear functional analysis, Springer, Berlin, 1985.

[4] Z. Ding and A. G. Kartsatos, P-regular mappings and alternative results for perturbations of $m$-accretive operators in Banach spaces, Topol. Meth. Nonl. Anal. 5 (1995), 291-304.

[5] D. E. Edmunds and W. D. Evans, Spectral theory and differential operators, Oxford Univ. Press, New York, 1987.

[6] D. E. Edmunds and J. R. L. Webb, Remarks on nonlinear spectral theory, Boll. Un. Mat. Ital. B (6) 2 (1983), 377-390.

[7] S. Fučik, J. Nečas, J. Souček and V. Souček, Spectral analysis of nonlinear operators, Lect. Notes Math. \#346, Springer, Berlin, 1973.

[8] M. Furi and M. Martelli, On $\alpha$-Lipschitz retractions of the unit closed ball onto its boundary, Atti Accad. Naz. Lincei, 57 (1974), 61-65.

[9] M. Furi, M. Martelli and A. Vignoli, Contributions to the spectral theory for nonlinear operators in Banach spaces, Ann. Mat. Pura. Appl. 118 (1978), 229-294.

[10] M. Furi, M. Martelli and A. Vignoli, On the solvability of nonlinear operator equations in normed spaces, Ann. Mat. Pura. Appl. 124 (1980), pp. 321-343.

[11] M. Furi and A. Vignoli, A nonlinear spectral approach to surjectivity in Banach spaces, J. Funct. Anal. 20 (1975), 304-318.

[12] M. Furi and A. Vignoli, Spectrum for nonlinear maps and bifurcation in the nondifferentiable case, Ann. Mat. Pura Appl. 113 (1977), 265-285.

[13] K. Georg and M. Martelli, On spectral theory for nonlinear operators, J. Funct. Anal. 24 (1977), 140-147.

[14] M. Martelli, Positive eigenvectors of wedge maps, Ann. Mat. Pura. Appl. 145 (1986), pp. $1-32$.

[15] R. H. Martin, Nonlinear operators \& differential equations in Banach spaces, Wiley, 1976.

[16] I. J. Maddox and A. W. Wickstead, The spectrum of uniformly Lipschitz mappings, Proc. Roy. Irish Acad. Sect. A, 89 (1989), 101-114.

[17] E. U. Taradfar and H. B. Thompson, On the solvability of nonlinear noncompact operator equations, J. Austral. Math. Soc. (Series A) 43 (1987), 103-126.

\section{Department of Mathematics}

University of SOUTH Florida

TAMPA, FL 33620-5700, USA

E-mail address: wfeng@chuma.cas.usf.edu 


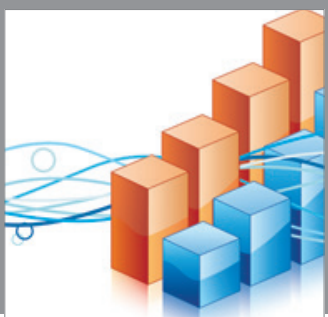

Advances in

Operations Research

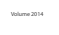

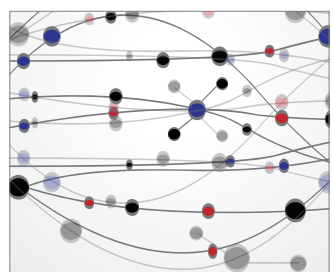

\section{The Scientific} World Journal
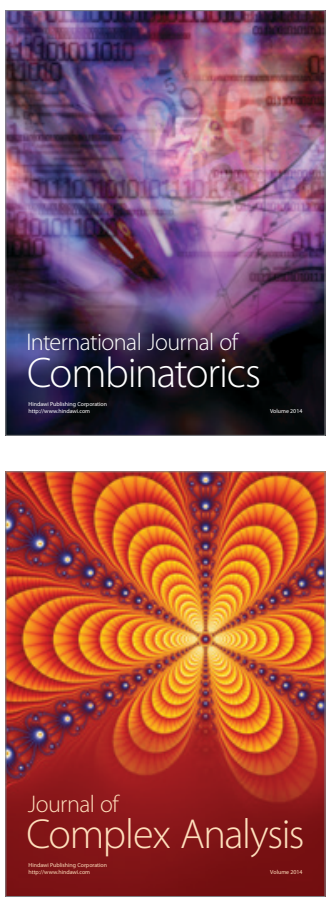

International Journal of

Mathematics and

Mathematical

Sciences
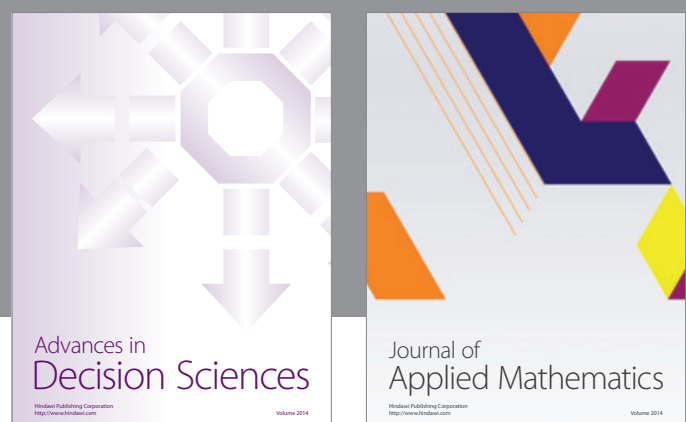

Journal of

Applied Mathematics
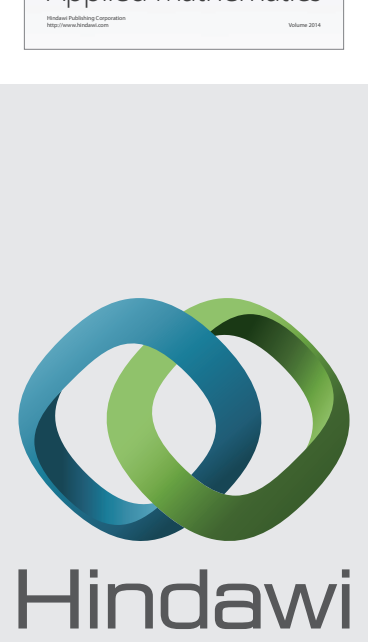

Submit your manuscripts at http://www.hindawi.com
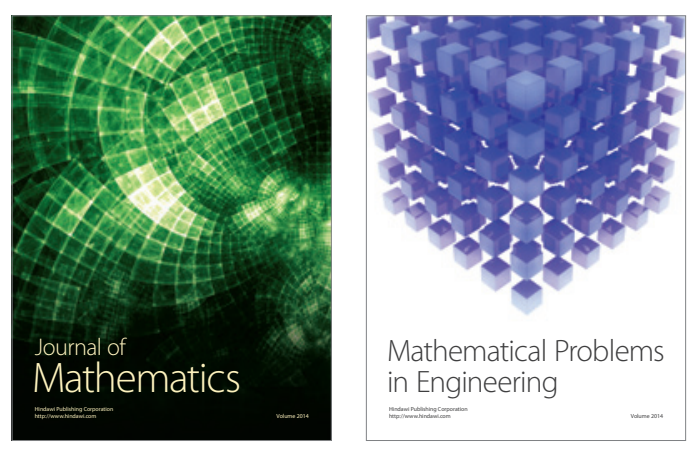

Mathematical Problems in Engineering
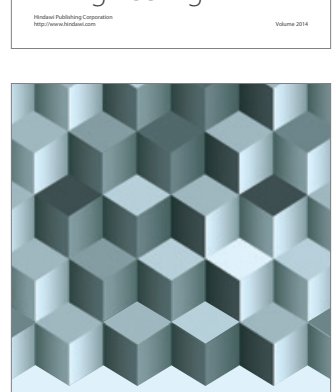

Journal of

Function Spaces
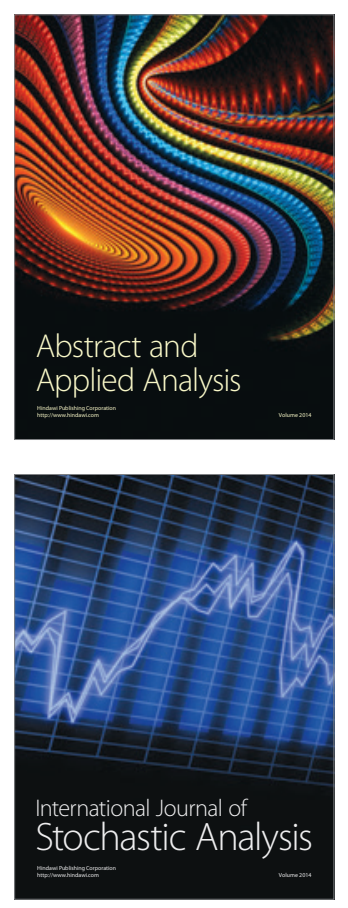

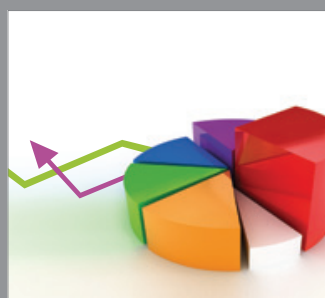

ournal of

Probability and Statistics

Promensencen
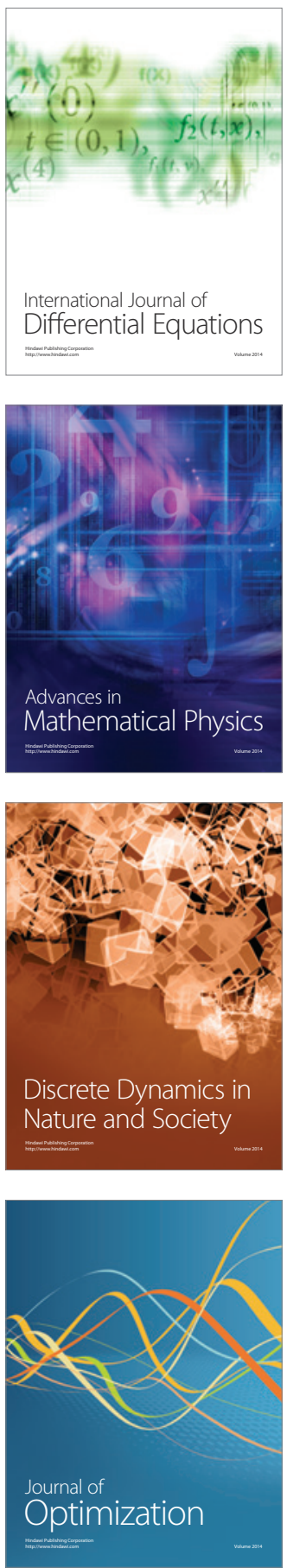